\title{
Is the Future Bright? The Potential of Lightboard Videos for Student Achievement and Engagement in Learning
}

\author{
Mark Lubrick ${ }^{1 *}$, George Zhou ${ }^{1}$, Jingsheng Zhang ${ }^{2}$ \\ ${ }^{1}$ University of Windsor, CANADA \\ ${ }^{2}$ Liaocheng University, CHINA
}

Received 28 November 2018 - Revised 21 February 2019 • Accepted 21 February 2019

\begin{abstract}
Lightboard technology has only been around since 2013, but has already shown up on numerous campuses worldwide. There is a dearth of research related to lightboard videos, so there is a need to systematically explore its potential and best practices. This paper explores the pedagogical potential of lightboards for higher education through theoretical analysis and relevant literature evidence. Using relevant theoretical frameworks, including Cognitive Load Theory, Cognitive Theory of Multimedia Learning, and Social Learning Theory, we argue that the lightboard technology may improve student achievement and learning engagement, since it displays an onscreen instructor, who has the possibility to utilize gestures. Papers that compared videos with and without onscreen instructors, as well as gesturing and no gesturing cases, are reviewed in terms of the impact on learning outcomes, cognitive load, and engagement and/or social aspects. The relevant literature did not, however, provide clear insight about the benefits that a lightboard video would provide. Therefore, we advocate for further empirical research directly studying lightboard videos. Relevant questions and directions for future research are identified.
\end{abstract}

Keywords: lightboard, onscreen instructor presence, student engagement, learning achievement, gesturing and signaling

\section{INTRODUCTION}

The purpose of this conceptual paper is to look at the potential of lightboard videos in terms of improving student achievement and engagement in learning. A lightboard is a large panel of glass with LED lights around the edges, which cause the markers to fluoresce on the board (Figure 1). It allows an instructor to create videos where they are on screen and can interact with multimedia materials, including writing directly on the board (see Figure 1). Using a switcher, instructors can combine multimedia (PowerPoints, images, videos, simulations, etc.) and gesture at or write directly on this media. Lightboard technology was originally developed in 2013 by Michael Peshkin, who shared the plans as open source hardware for others to build. Given its convenience for the instructor's presence in the video, the lightboard has shown up on more campuses worldwide in the past several years. Naturally, questions arise with regards to the potential of this technology and best pedagogical practices. There is currently little available literature on this aspect. Those articles that do exist either discuss how to design/modify the lightboard (Skibinski, DeBenedetti, Ortoll-Bloch, \& Hines, 2015) or how it has been used (Fung, 2017). There appears to be no empirical studies regarding its effectiveness. This paper aims to identify what key features of lightboard videos may impact its effectiveness and provide recommendations for future research around this new technology. Arguments will be made through an analysis of relevant theories and literature that are closely relevant to the exploration of the impacts of an instructor's presence and performance in videos.

(c) 2019 by the authors; licensee Modestum Ltd., UK. This article is an open access article distributed under the terms and conditions of the Creative Commons Attribution License (http://creativecommons.org/licenses/by/4.0/). \mlubrick@uwindsor.ca (*Correspondence) \gzhou@uwindsor.ca $\square$ zhangjingsheng@lcu.edu.cn 


\section{Contribution of this paper to the literature}

- As far as the authors are aware, this is the first literature review that discusses the lightboard or relates it to theory.

- Provides a theoretical framework of why lightboard videos could be useful for engaging students and improving knowledge retention.

- Provides suggestions for future research directions.

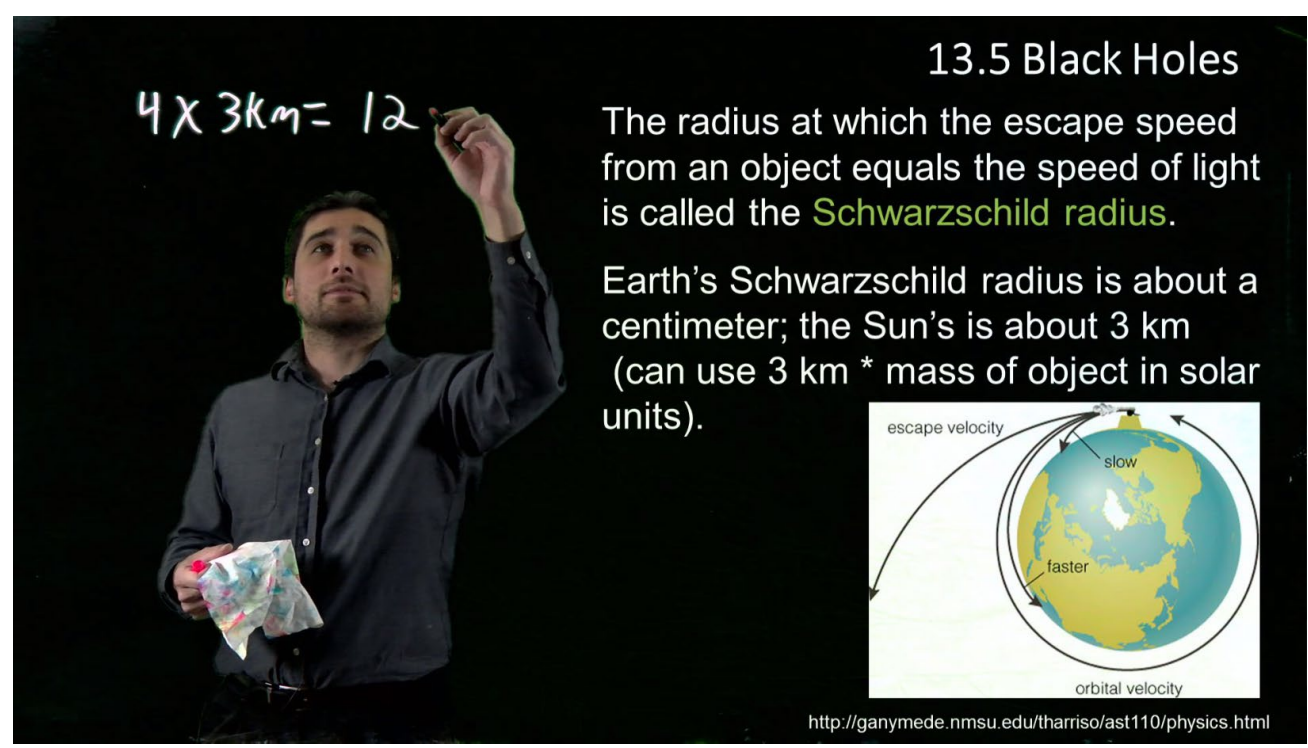

Figure 1. Example of a Lightboard Video

\section{Search Methods}

For this literature review, peer reviewed articles and dissertations were included. There were three databases searched: ERIC, PsycINFO, and the Leddy Library online search catalogue. The search looked for articles that included the keyword "video*" (to allow for videos, as well) in the title and one of the following keywords anywhere in the article: engagement, instructor presence, learning, gesture, knowledge, retention, cognitive theory of multimedia learning, and social agency theory. It is important to note that any phrases were not search strings requiring the full phrase to be used (i.e. cognitive and video would have come up). In addition, separate searches in the three databases looked for the following keywords: lightboard*, cognitive theory of multimedia learning, social agency theory. Finally, any article that was found had the mentioned references searched to identify more potential articles.

When articles were found, they were vetted by fairly stringent criteria. The articles needed to focus on asynchronous videos, not any sort of webconferencing. The videos had to be instructor created (possibly with assistance from others, such as instructional designers), not student created. Virtual reality or 360-degree videos were not included. The focus was not on network factors (such as bandwidth). The studies had to compare two or more videos in terms of gesturing or onscreen agents, except for papers that provided relevant theory. Experimental papers needed to include measures of engagement, or learning, or cognition. Self-reported estimates of learning were not included. For gesturing studies, the focus was on gestures that matched the speech. For onscreen agent studies, papers had to include at least one case where there was a live person used. Studies that only considered animated pedagogical agents were not included.

\section{THEORETICAL ANALYSIS}

\section{Cognitive Load Theory}

Cognitive Load Theory (CLT) makes an important distinction between biologically primary and secondary knowledge. Biologically primary knowledge is knowledge that we have evolved to know and should be easy to acquire with minimal cognitive effort. This knowledge may be leveraged to help acquire biologically secondary knowledge, which must be explicitly learned through deliberate and conscious effort. "Our ability to obtain 
biologically secondary information from other people via imitation and listening is itself a biologically primary task that does not need to be taught" (Paas \& Sweller, 2014, p. 31). Students should be able to learn from watching an instructor without putting extra cognitive strain into thinking of how best to learn by watching, as they would likely learn this skill easily and early on. Of course, this does not consider if other media is displayed at the same time.

The aim of instruction is to have knowledge transferred into long term memory, as "most human cognitive activity is driven by information held in long-term memory" (Paas \& Sweller, 2014, p. 30). The problem is that knowledge must first be stored in working memory, which has a limited capacity for how much information can be stored (roughly seven elements) and for how long (roughly 20 seconds). When we learn new material, we try to store the important elements in working memory, relate it to knowledge in long term memory, and ultimately integrate it into long-term memory. Paas and Sweller report that information brought from long term memory into working memory does not have the same limited capacity as new biologically secondary information. Thus, a learner will store progressively more information in long-term memory and understanding takes place when all of the relevant aspects can be processed together in working memory. With respect to multimedia representations, pictorial, verbal, or written knowledge could all be stored in long-term memory.

CLT defines three types of cognitive load (Paas \& Sweller, 2014). Intrinsic cognitive load is related to the cognitive effort required to acquire the biologically secondary knowledge. The amount of intrinsic cognitive load is determined by the level of "element interactivity", which can be roughly thought of as the inherent complexity of the material. Extraneous cognitive load is cognitive load that is being used for knowledge not directly useful for the task at hand. This can be caused by improperly designed instruction with elements that might distract from the main knowledge to be acquired. Germane cognitive load "refers to the working memory resources devoted to intrinsic cognitive load minus the resources devoted to extraneous cognitive load" (Paas \& Sweller, 2014, p. 38). It is a measure of how much cognitive load is being used effectively. As previously noted, cognitive capacity is limited. If more capacity is devoted to the extraneous cognitive load, it will diminish how much is available for intrinsic cognitive load. If the amount of information to be learned exceeds the cognitive load capacity, effective learning will not take place. Thus, it becomes essential to reduce extraneous cognitive load. Since the lightboard allows the instructor to show up in the video, it is essential to determine if the presence of an instructor in a video would increase the extraneous cognitive load.

\section{Cognitive Theory of Multimedia Learning}

The Cognitive Theory of Multimedia Learning (CTML), as defined by Mayer (Mayer, 2005; Mayer, Heiser, \& Lonn, 2001; Mayer \& Moreno, 2003) and expanded in several subsequent works (Brame, 2016; Ginns, 2005; Mayer, 2017; Schneider, Beege, Nebel, \& Rey, 2018), is of particular relevance when considering the features of the lightboard and their potentials. It has been used by scholars as a basis for providing design advice for instructional videos (Brame, 2016). CTML agrees with CLT that humans have limits on their cognitive processing; however, it points out that, "humans possess separate information processing channels for visually/spatially represented material and auditorily/verbally represented material" (Mayer, 2005, p. 47). Each of these channels does have a limited capacity, but their limits function separately, although it is worth noting that information that enters via one channel may possibly be converted to be processed in the other channel. In the process of active learning, people must first pay attention to the incoming information to allow it to enter sensory memory via eyes and ears. The information can only be stored in sensory memory for a very brief time. People must then pick the relevant parts to bring into working memory. Since humans have dual channels for processing, they will be picking separate words and images. Within working memory, these selected bits of information can be temporarily manipulated to create separate verbal and pictorial models, respectively. Afterwards, these two models will be combined with each other and with prior knowledge taken from long-term memory. Finally, this new knowledge could be encoded into long-term memory. Once the knowledge is encoded into long-term memory, it can be combined with future models. This process does not have to occur in the linear manner described, it can be iterative. It is also a repetitively occurring process throughout the learning, as only limited pieces can be processed at a time.

Similar to CLT, CTML defines three types of cognitive processing. Extraneous processing is the cognitive process that does not support the learning and is likely caused by a poor design. Essential processing refers to representing the material in working memory. It is determined by the complexity of the material. Generative processing "refers to cognitive processing aimed at making sense of the presented material and is caused by the learner's motivation to learn" (Mayer 2005, p. 60). These three processes all draw from the limited cognitive capacity that an individual has, so that if extraneous processing is too large, it will detract from essential and generative processing. Thus, similar to CLT, extraneous processing must be controlled. Learners need to be motived for generative processing to occur. To consider the effectiveness of lightboard technology, it is important to determine whether the instructor presence will increase extraneous processing. Even if it does, will it possibly motivate students to learn? Does one of these effects out-weight the other? 
Mayer (2017) proposes 12 principles for CTML. Among these principles, the multimedia principle states that people learn better from words and pictures than from words alone. It is obvious that the lightboard allows both words and pictures/diagrams to be displayed. Butcher concluded that, "diagrams should be no more complex than necessary to communicate key concepts" (2014, p. 183). The lightboard allows instructors to draw out simple diagrams, while using words to describe the drawing or the process of its creation.

The coherence principle states that instructors should eliminate any extraneous material from the lesson. It is meant to reduce extraneous processing by eliminating so called 'seductive details', which are interesting, but irrelevant pieces of information. Mayer (2017) found this principle to be supported by 12 out of 12 experiments and yielded a medium-to-large effect size. An interesting boundary condition was that, "adding emotionally interesting details to essential elements in a graphics on viruses (such as giving the virus and host cell human-like facial expressions) improved test performance (Mayer \& Estrella, 2014; Plass, Heidig, Hayward, Homer, \& Um, 2014; Um, Plass, Hayward, \& Homer, 2012)" (as cited in Mayer, 2017, p. 407). This principle and boundary condition are of particular relevance to the use of the lightboard. There is the worry that adding an instructor in the video will create a 'split-attention effect', whereby the student divides the visual cognitive processing between the instructor and whatever text and graphics are on the screen. This could cause a cognitive overload due to extraneous processing. That said, it could be argued that the instructor would likely be an 'emotionally interesting detail', especially since the boundary study focused on anthropomorphizing a virus. This also begs the question whether there would be anything that the instructors could do to make their presence in the video more 'emotionally interesting'.

The signaling or cueing principle is meant to guide essential processing. It involves using vocal emphasis to highlight key terms and can also "involve highlighting relevant portions of the graphics using features such as arrows, colouring, or spotlights" (Mayer, 2017, p. 407). Mayer found that 18 out of 20 experiments supported this with a median effect size of 0.46 . He also stated that a boundary condition was that the signaling principle had been shown for students with low prior knowledge, but not for students with high prior knowledge. A recent metaanalysis of 103 studies related to signaling did find a contrasting result, stating that, "learners' domain-specific knowledge does positively influence the effectiveness of signaling techniques" (Schneider et al., 2018, p. 19). Schneider at al. expanded the definition of signaling to include gesturing effects, although most of the gesturing studies they looked at related to animated pedagogical agents, not live people. They found that learners' retention and transfer performance both appeared to be positively impacted by signalling. Schneider et al. argued that signaling does require extra cognitive processing and it was most effective in situations where the learning was not controlled by time limits. When looking at specific signaling behaviour, they did find that, "graphical signaling with pointing gestures reached significance as a moderator of retention knowledge, but not as a moderator for transfer knowledge", and that, "color improves learning in both pictorial and textual cue modes and might enhance the aesthetics of an environment and foster emotional and motivational variables that mediate higher learning outcomes" (Schneider et al., 2018, p. 20).

Signaling and gesturing are naturally facilitated by the lightboard. The lightboard can mix multiple medias, so that any normal animations or signaling would be possible. It also allows for natural signaling from the instructor. Since instructors have multiple coloured markers with the lightboard, they can use colour to highlight or emphasize key points. As signaling was shown to decrease cognitive load (Schneider et al., 2018), an instructor utilizing this strategy may also be able to help mitigate any potential increase in cognitive load that their presence may cause.

The spatial contiguity principle states that instructors should place printed words next to the corresponding graphics. This is meant to limit extraneous processing that would result from having to scan back and forth to see what words relate to which part of a graphic. By putting the words close to the graphic, it signals to the learner where to look. Mayer (2017) found this principle to be supported by 8 out of 8 experiments, resulting in a large effect size. The lightboard allows an instructor to draw words close to a graphic. The physical process of writing the word and the ability to use different colours to draw attention might create an increased signal to the learners.

The temporal contiguity principle states that narration should occur simultaneously with the corresponding graphic. This is meant to decrease extraneous processing, as compared to presenting the narration and graphic at separate times. Mayer (2017) stated, "successive presentation will create massive extraneous processing because you have to keep the entire narration in working memory until the graphics are presented, or vice versa, leaving less cognitive capacity for making sense of the incoming material." The contiguity principle was supported by 8 out of 8 experiments for a large effect size. The boundary conditions were that this principle does not apply for material presented in small chunks or if the material is simple for the learners. The lightboard naturally lends itself to discussing a figure while it is on screen, so the instructor can interact with it. Not only are instructors likely to discuss the image while it is on the screen, they can also use annotations, gestures, or other signals. For any graphs, instructors can talk while drawing them out.

The modality principle states that instructors should use spoken text rather than printed text with graphics. This is meant to avoid a split attention affect for learners where they cannot focus on the words and graphics at the same time. Mayer (2017) found this was supported by 42 out of 51 experiments for an effect size of 0.72 . An earlier 
meta-analysis also found that, "students who learned from instructional materials using graphics with spoken text outperformed those who learned from a graphics with printed text" (Ginns, 2005, p. 326). Further, Reinwein (2012) argued that the modality principle may be strongest for system-paced lessons rather than learner-paced, dynamic graphics rather than static, and transfer tests rather than retention tests. The modality principle is interesting to our discussion of the lightboard for a number of reasons. Firstly, the lightboard should naturally facilitate the use of spoken word, as the instructor is on the screen and will likely have less room for text to appear. It is also interesting to consider if adding the instructor will create the very split attention effect that the modality principle is trying to avoid. Will the students be able to focus on the graphic with the instructor present? Are there strategies, such as gesturing, that can be used to guide the learners' attention? These are questions that will be reviewed in later sections.

\section{Social Agency Theory}

Parts of CTML focus on generative processing and are referred to as Social Agency Theory (SAT). SAT emphasizes the social aspect of learning because "when the learner feels social partnership with the instructor, the learner will exert more effort to understand what the instructor is saying, which results in better learning outcomes" (Mayer, 2017, p. 414). Therefore, when considering the significance of media, we should ask, will the technology increase the sense of partnership between learners and the instructor?

SAT states that people will learn better from a standard accented human voice than from a machine-like voice. While the voice used in videos is often a design choice, it is natural for lightboard videos to include a human voice, as the instructor is already on the screen. In addition, Mayer states that, "a human voice speaking with a standard accent conveys a sense of social presence - that is, it conveys the idea that someone is speaking directly to you" (2014, p. 351). The lightboard has the potential to convey the feeling that an instructor is speaking directly to a student, as the instructor looks directly into the camera. SAT also states that people will learn better when the words in a presentation are in a conversational style rather than a formal style. It suggests an instructor to utilize "you" and "I" rather than third-person constructions and make direct comments about themselves and their own experiences. The lightboard can easily achieve this since instructors are on the screen and thus, are more likely to reference themselves.

"Humanlike gestures, facial expressions, eye gaze, and movement can serve as a social cue" (Mayer, 2014, p. 363). An onscreen agent (animated or human) is considered high-embodied when it draws while talking or employs gesture, facial expression, and eye gaze. In contrast, if an onscreen agent stands motionless in contrast to how humans usually behave, it is low-embodied. SAT states people learn better from high-embodied agents rather than low-embodied agents, which may be because they will try harder with high-embodied agents. The lightboard would naturally have a high-embodied onscreen agent, utilizing talking and facial expressions, as it is a real person who is on the screen. To utilize the lightboard to its full potential, instructors would also likely incorporate gestures, drawing, and eye gaze. Conversely, Mayer (2014) worries that an onscreen agent would be a seductive detail that would increase the cognitive load and mitigate any benefits from the social cues. It is important to note that the argument was based on animated pedagogical agents.

\section{EVIDENCE FROM LITERATURE}

There already exists "an agreement among researchers that video based learning in conjunction with appropriate pedagogical methods has the potential to improve the learning outcome" (Yousef, Schroeder, \& Chatti, 2014, p. 114). Therefore, this paper is not interested in comparing learning between videos and other media; instead, its arguments are more based on comparison between various video formats. Scholars often consider two aspects of learning experiences when talking about the impacts of video media: learning achievement and learning engagement. There are two types of learning achievement: retention (recalling facts) and transfer (being able to apply that knowledge to a problem that has the same deep structure, but different surface structure). The study of media impacts on learning achievement often takes an experimental research design to compare the gains between treatment and control groups right after the invention or through a follow-up test (Church, Ayman-Nolley, \& Mahootian, 2004; Cook, Duffy, \& Fenn, 2013; Hoogerheide, Loyens, \& van Gog, 2014; Valenzeno, Alibali, \& Klatzky, 2003; van der Meij, 2017; van Wermeskerken, Ravensbergen, \& van Gog, 2017; Yeo, Ledesma, Nathan, Alibali, \& Church, 2017).

Measurement of media impacts on learning engagement is more complicated. One of the most common proxies for engagement is to use video view times (Bhat, Chinprutthiwong, \& Perry, 2015; Guo, Kim, \& Rubin, 2014; Wang \& Antonenko, 2017), with some studies also considering re-watched parts of the video (van der Meij, 2017). Eye tracking software has been used to track learners' engagement with videos (Pi \& Hong, 2016). Some studies look at the completion of a post video quiz (Guo et al., 2014; Wang \& Antonenko, 2017) or learners' participation in a 
discussion forum shortly after viewing a video (Bhat et al., 2015). Some studies have used surveys to have students self-report their attention/engagement level (Pierson, 2017). Each approach has its own limitations.

\section{Onscreen Instructor Presence in Videos}

\section{General considerations}

One of the most significant differences between lightboard videos and other types of videos is the presence of a real instructor. Therefore, to conceptualize the impacts of lightboard technology, it is crucial to look at videos that involve an instructor being visible in the video. The review will include studies that compare cases with or without an instructor and studies that compare different methods of having the instructor on screen.

It is important to define the types of videos that involve an instructor presence since scholars have used different terms in the literature. For example, one study used the term "talking head" (Ilioudi, Giannakos, \& Chorianopoulos, 2013) for what another referred to as "classroom capture" (Pi \& Hong, 2016). For this paper, classroom capture refers to videotaping a traditional lecture, such that the instructor is in frame beside a board or presentation. A talking head video refers to a close up (shoulder and face) of an instructor looking at the camera. Hands may or may-not be visible in these types of videos and this is often not specified in the studies. A picture-in-picture video refers to a presentation with an image of the instructor included in the corner. Finally, green screen videos are those where the instructor is in front of, and embedded in, the presentation and the instructor is able to interact with the presentation.

How will an instructor presence influence student attention? This question is critical for deciding on including an instructor in a video or not. Ayres and Sweller (2014) argue that the instructor, and particularly the instructor's face, is likely to attract students' attention, resulting in a kind of 'split attention effect', as learners must divide their attention between the instructor and the demonstration. In the cases of low-embodied animated pedagogical agents, Mayer stated, "that people do not necessarily learn more deeply from a multimedia presentation when the speaker's image is on the screen rather than not on the screen" (2014, p. 346). Paas and Sweller (2012), however, argued that because humans have presumably evolved to observe and imitate the behaviors of others, learners may be able to actively interpret the actions of a model without the risk of cognitive overload. In other words, watching is not a cognitively demanding task since it fits to the domain of biologically primary knowledge. If the social cues from having an instructor on screen can help generative processing without causing undue extraneous processing, then learning should be positively impacted.

\section{Learning outcomes}

Among the studies that explicitly looked at videos with or without the instructor present, three studies compared a picture-in-picture video with an equivalent video without the instructor (Kizilcec, Papadopoulos, \& Sritanyaratana, 2014; Moreno et al., 2001; Wang \& Antonenko, 2017). These three studies measured both retention and transfer results. Kizilcec, et al. and Moreno et al. did not find a significant difference on retention and transfer between groups who used videos with an instructor on screen and those without. Wang and Antonenko had "easy" and "hard" questions for their measurement of both transfer and retention. They found that there was greater recall of the easy retention questions for the video with the instructor as compared to the video without, although the rest of their results had no significant difference. A study used classroom capture versus a "Khan-style" video whereby, the steps were written out, but the instructor was not onscreen (Ilioudi et al., 2013). The study was conducted in three separate learning modules. For the first two modules, there was no significant difference on a learning test, but in the third module, which they deemed the hardest, the instructor present group did significantly better than the Khan-style group. Two studies compared a green screen video to a video without the instructor (Hoogerheide et al., 2014; van Wermeskerken et al., 2017). Van Wermeskerken et al. found no difference on retention or transfer tests. Similarly, Hoogeridge et al. did not find significant difference for retention and transfer; however, they did find that far transfer results were statistically significantly better for the group with the instructor.

There were three experiments that looked at comparing videos with and without the instructor's face present (van Gog, Verveer, \& Verveer, 2014; van Wermeskerken \& van Gog, 2017; van Wermeskerken, Grimmius, \& Gog, 2018). To create the "no face" condition, the researchers simply cut off approximately the top half of the video (cutting out the face, but leaving the body and hands that were performing the task). Thus, the two conditions were not equal in terms of the display size and there would be concerns that the "no face" condition might not seem authentic. Van Wermeskerken \& van Gog (2017) and van Wermeskerken et al. (2018) found no difference between groups on the knowledge test or building task tests. In the study of Van Gog et al. (2014), after the second viewing, the "with face" group performed better on the building task than the "no face" group. There were three studies where the exact methods of instructor presentation were difficult to determine (Dey, Burn, \& Gerdes, 2009; Hong, Pi, \& Yang, 2018; Pi \& Hong, 2016). Dey et al. compared videos with and without the instructor and found no 
difference in transfer or retention. Hong et al. looked at the impact of instructor presence in two types of videos meant to teach either declarative or procedural knowledge. They found that for procedural videos, there was no difference, while for declarative videos, the instructor present group did significantly better on a post test. Pi and Hong looked at four video conditions with either just the instructor, just the PowerPoint, classroom capture, or instructor and PowerPoint. They found the instructor and PowerPoint group had the highest results and the instructor only group had the lowest, with the differences between groups being significant. It was not clear how the instructor showed up in videos and there was no information about whether the PowerPoints had essential information for the learning which might account for the deficit in the instructor only condition.

From all of these results, it is hard to draw any significant conclusions. The experimental types and results were quite mixed. It is worth noting that for all of the experiments that compared two videos where the only difference was the presence of the instructor, none of them found that the instructor's presence negatively impacted the learning outcomes. Of course, only a few found positive gains.

There were also three studies that compared different forms of having the instructor on screen. Chen and $\mathrm{Wu}$ (2015) used lecture capture, green screen, and a modified picture-in-picture that involved a third window showing a table of contents (this case had the smallest video of the instructor and the video was not directly on the slides). They found that the picture-in-picture method yielded lower test scores than the other two groups. A study comparing classroom capture to an experimental design showing the PowerPoint with a title of the main idea below, a window with the instructor as a talking head with their name below, and a video window showing students reacting, found the experimental design had higher scores than the classroom capture (Pao-Ta Yu, YuanHsun Liao, \& Ming-Hsiang Su, 2013). It is important to note that the PowerPoint slides were shown more clearly in the experimental design than the classroom capture, which could have impacted the results. Kizilcec et al. tried to develop a novel approach whereby the instructor's face was shown on screen only sometimes in the hope that, "the presence of his or her face would act as a cognitive aid to direct learners' attention to relevant content during instruction" (2015, p. 725). They did not find a significant difference between the groups for learning outcomes. It would be difficult to draw any conclusions from such a small and diverse set of experiments.

\section{Cognitive load}

It is essential to consider cognitive load. Will the instructor's presence create a split-attention effect or otherwise increase cognitive load through extraneous processing? Is it possible that watching the instructor onscreen is a sort of biologically primary knowledge that will not require extra cognitive processing? Could benefits such as social cues counteract any cognitive increases from having the instructor on screen?

Wang and Antonenko (2017) used picture-in-picture for "easy" and "difficult" videos compared against videos without the instructor, and estimated cognitive load. For the easy video there was no difference, but for the difficult videos, the group with the instructor present reported lower mental effort. Hoogerheide et al. (2014) found there was no difference in self-reported mental effort between the green screen video and the equivalent without the instructor. Chen and Wu (2015) found for students classified as "visualizers" that the cognitive load for their modified picture-in-picture was significantly higher than for lecture capture or green screen. Hong et al. found no difference in mental effort for the declarative videos, but for the procedural videos, "the participants who watched the video without the instructor reported significantly less cognitive load" (2018, p. 78). It is worth reminding the reader that for these declarative videos, the instructor present group had higher learning scores and for the procedural videos, there was no difference. Thus, the learners were able to get the same procedural knowledge for less reported mental effort and more declarative knowledge for the same reported mental effort, when the instructor was onscreen. Finally, in the study from Kizilcec et al. (2015), based on self-reported cognitive load, their strategic video where the picture of the instructor was only there sometimes was actually more cognitively demanding than the video with the instructor there at all times. While is hard to generalize from this relatively small and diverse set of articles, there does seem to be a support for the fact that the instructor's presence does not increase cognitive load, and in fact, may actually decrease it. It is essential to note that these studies used selfreported values of cognitive load, which may have been inherently flawed.

\section{Engagement and attention}

This section will look at how incorporating an onscreen instructor influences the viewing habits and engagement of a learner. Similar to the learning outcomes section, this will start by looking at studies that compared videos with and without an instructor onscreen, then move to studies about the types of instructor presence onscreen. For picture-in-picture videos, Kizilcec et al. (2014) and Wang and Antonenko (2017) uses eye tracking data to study students' engagement with videos. Kizilcec et al. found that participants spent $41 \%$ of the time looking at the instructor's face and made transitions between the face and slide every 3.7 seconds, but as previously noted, there was no difference in learning outcomes for the groups. The participants in the study by Wang and Antonenko 
spent far less time looking at the instructor (26\% for easy topics and $22 \%$ for hard topics), though this could be because the window for the instructor only took up 7\% of the screen. Pierson (2017) did not use eye tracking data, but instead had students self-report their attention and whether they had been multitasking while watching the video. The participants were prompted by questions during the video and they also self-reported after the video, but there was no significant difference between the groups.

Van Wermeskerken et al. (2017) studied green screen videos where the instructor stood beside the slides, but did not interact with the slides. They used eye tracking software to compare the instructor group with the no instructor group in terms of how many referenced objects (i.e. mentioned verbally) learners focused on and if they focused on them quickly. While the time to focus was not different, the amount of fixations on referred items was lower for the instructor group, which led the researchers to conclude that, "an instructor who is visible in the video (and staring straight into the camera) may distract students' visual attention from what he is talking about" (van Wermeskerken et al., 2017, p. 7). It is worth reiterating that there was no difference between the groups in terms of retention or transfer tests. Pi and Hong (2016) looked at eye tracking data for the instructor and PowerPoint group and had the most extreme results reported at " $62.30 \%$ of the time fixating on the instructor, and $37.70 \%$ fixating on the PPT slides, on average" (2016, p. 140); however, as mentioned, they found that the instructor and PowerPoint group had the highest learning outcomes. Therefore, they concluded the presence of an instructor facilitated the enhanced learning outcome. In summary, it is noteworthy that despite the increased focus on the instructor in these studies, there was no decrease in learning outcomes, and in some cases, there was even an increase.

There were a few other studies that are unique enough that they should be interpreted separately from the above studies. Korving, Hernández, and De Groot (2016) compared three video cases with either just a large PowerPoint, a large PowerPoint and small instructor, or a small PowerPoint and large instructor. Participants were asked to watch one of the videos and report their level of attention. They then watched a different video type and reported their level of attention as compared to the previously watched video. Ultimately, they found no difference between having the instructor on the screen or not. For the three studies mentioned above that compared videos with or without the instructor's face (van Gog et al., 2014; van Wermeskerken \& van Gog, 2017; Wermeskerken et al., 2018), researchers used eye tracking data to compare students' learning experience. All three studies reported that the "no face" group spent more time focused on the task area, which is hardly surprising.

There were three studies that looked at attention/engagement with respect to different types of instructor onscreen videos (Bhat et al., 2015; Chen \& Wu, 2015; Ozan \& Ozarslan, 2016). Ozan and Ozarslan examined the rates at which resource videos were fully watched comparing green screen, talking head, and interview videos. Watching videos was not obligatory in their course. They found that interview videos were watched fully at $25.6 \%$ of the time, followed by talking head videos at $17.9 \%$, and green screen at $15 \%$. Chen and $\mathrm{Wu}$ investigated the sustained attention and emotion through measuring human brainwave signals and heart rate variability patterns. They found that the modified picture-in-picture video had significantly higher sustained attention than the green screen video. Suma Bhat et al. (2015) allowed students to pick between green screen or picture-in-picture videos, estimating their engagement based on total view times and whether they participated in a relevant discussion within 30 minutes of watching a video. Ultimately, they concluded that the green screen videos were preferred and appeared to be more engaging. It does not appear that the results of these studies overlap or that any general trends can be found.

\section{Social aspect}

Theoretically speaking, an instructor in video will help learners build a sense of relationship with the instructor. Pierson (2017) attempted to study this directly with a Student-Instructor Relationship Scale (SIRS). He found that the mean was higher for participants who saw the instructor's face within the video. Dey et al. found that participants who watched the personalized videos "disagreed with the notion that the instructor's image was distracting" (2009, p. 387). Kizilcec et al. (2014) had students rate different segments of a video and found that participants' ratings strongly favoured the segments showing the instructor's face. Since one of the main purposes of including the instructor in videos is to enhance generative processing, the fact that students are more positive towards this lecture format may contribute to their motivation. As a prelude to the next section, it is worth looking at the study of Suma Bhat et al. (2015) again for their explanation about why students preferred the green screen videos to the picture-in-picture:

We hypothesize that the modes primarily differed in their ability to make the instructor's gaze and gestures more directly accessible to learners and that the mode that offered more access to instructor's gestures and eye-gaze was probably the preferred mode by the vast majority of learners. We also hypothesize that these users, possibly owing to the resulting positive affect created by improving the instructor's social presence, showed more engagement with the videos (via larger watch times), preferred the streamed mode of 
viewing videos (indicating immediacy in user response) and covered a larger proportion of lectures (p. 312).

\section{Gesturing in Videos}

\section{Gestures in general}

There are many kinds of gestures. A beat gesture is generally a quick stroke of a hand or fingers through the air and is "usually thought to be nonnarrative, motorically simple, rhythmic gestures that [does] not convey semantic content related to speech" (Kang, Hallman, Son, \& Black, 2013, p. 827). A representational gesture is meant to "depict a spatial or motor referent by pantomiming a particular action, by demonstrating a spatial property, or by creating such a referent for an abstract idea" (Hostetter, 2011, p. 298). In some cases, the representational gesture is further divided into metaphoric gestures, where the gesture depicts abstract referents or ideas metaphorically without the hand motion relating directly to the information, and iconic gestures whereby, "a specific gesture bears a close formal relationship to the semantic content of speech" (Kang et al., 2013, p. 827). Finally, dietic gestures are where the gesture refers to an object or region of space directly. Gestures can also be classified based on how they relate to the information to which the gesture is tied. A redundant gesture does not add any new information; it merely points to or emphasizes an idea. A nonredundant or supplementary gesture will provide new information that is not provided otherwise (i.e. a gesture that conveys information not contained in a verbal description). Finally, a mismatching gesture is one where the gesture contradicts the information being presented (i.e. pointing right when saying left).

There are a number of theoretical reasons to think gestures could benefit learners. Based on the information packing hypothesis, "representational gestures help organize visuo-spatial knowledge into a series of discrete units" (Kang et al., 2013, p. 827). This would decrease the cognitive load of a viewer. This may be because gesturing can serve a similar role to cueing in terms of managing essential processing; however, Pi et al. (2017) advocate that gestures can be more effective since, "learners need fewer working memory resources to follow the instructor's pointing gestures than nonhuman cues" (p. 1021-1022). Another potential benefit is that since "gestures provide motoric information, they also add another modality, which might have positive effects on learning (i.e. a modality effect)" (Ouwehand, van Gog, \& Paas, 2015b). Ouwehand et al. (2015b) further state that the motoric information is automatically processed, which does not add load to the limited capacity of working memory. If gestures can indeed reduce cognitive effort and act as another modality to enhance learning without requiring extra working memory, they pose a huge potential benefit for implementation in lightboard videos. A meta-analysis found that 54 out of 63 samples had positive effect sizes for gesturing and concluded that gestures do significantly improve communication (Hostetter, 2011).

\section{Learning}

Twelve studies were found that compared a group that watched gesturing in videos against a control group which had an equivalent video, but no gesturing was used. Seven of them found that the gesturing group did significantly better for learning gains than the control group (Carlson, Jacobs, Perry, \& Breckinridge Church, 2014; Church et al., 2004; Cook et al., 2013; Koumoutsakis, Church, Alibali, Singer, \& Ayman-Nolley, 2016; Pi, Hong, \& Yang, 2017; Rueckert, Church, Avila, \& Trejo, 2017; Valenzeno et al., 2003). Three studies found no significant difference between the groups (Fiorella \& Mayer, 2016; Ouwehand et al., 2015b; Ouwehand, van Gog, \& Paas, 2015a). One found that the gesture group did worse than the control group (Yeo et al., 2017). One had mixed results (Kang et al., 2013). Yeo et al. (2017) recognized that the gestures in their study were too redundant, as the instructor merely pointed at the object they were describing, and thus, did not add information. These redundant gestures may have actually distracted the students from looking at the object in question. Similarly, Kang et al. (2013) looked at both representational gestures and beat gestures, and compared the performance of a group who watched those gestures against a no gesture group. The representational group scored the highest, while the beat gesture group scored the lowest. Thus, the representational gestures seemed to help with learning, but, "it is possible that listeners with beat gestures lost the chances to acquire semantic information once they [payed] attention to a speaker's beat gestures, because it possibly [limited] their mental resources" (p. 834-835). One serious limitation of the Kang et al. study was that they did not perform a pretest and thus, did not control for different levels of prior knowledge between the groups. All three studies that found no significant difference between gesture and no-gesture groups also used pointing gestures to indicate specific regions (Fiorella \& Mayer, 2016; Ouwehand et al., 2015b, 2015a). Cook et al. (2013) examined participants' performance on an immediate retention test, a retention test 24 hours later, and a transfer test 24 hours later. They found that the gesture group outperformed the no-gesture group on all three tests. Furthermore, even if the difference in performance on day one was considered as a covariate, the gesture group still had a significantly higher gain on the delayed test, as compared to the no gesture group. They 
concluded that, "gesture affects initial learning, and additionally affects how learning is consolidated over time" (Cook et al., 2013, p. 1867). Overall, these studies seem to offer support to a conclusion that gesturing can support learning gains over no gesturing conditions. An important caveat appears to be in relation to redundant pointing gestures or beat gestures. These may not improve the learning and in some cases, can actually have a negative impact on learning.

\section{Cognitive load}

Only three of the studies compared cognitive load between gesture and no-gesture groups (Ouwehand et al., 2015b, 2015a; Pi et al., 2017). In all three studies, a scale adapted from Paas (1992) was used to measure mental effort while working on the problems. These studies did not find significant difference in cognitive load between the gesture and no gesture groups. This is a bit of a surprising result, as it was hoped that gestures would reduce cognitive load. Since the presence of an instructor would not increase cognitive load (in some cases may even decrease it), as concluded in previous section, it seemed likely that including gestures would positively impact essential processing, such that cognitive load would be decreased. It may seem to contradict the idea that gestures might be automatically processed without extra demands on working memory. Of course, more thorough research would be required than just three studies.

\section{Engagement due to socialization or attention grabbing?}

Four studies investigated learners' engagement or attention associated with gestures. Among them, Valenzo et al. (2003) recorded how often a child turned their head away from the video lesson and found that the no gesture group turned away significantly more often. Ouwehand et al. (2015a) and Pi et al. (2017) used eye tracking equipment and found that gestures caused the learners to focus more on the relevant task areas. Ouwehand et al. (2015b) explored the time on task and found that for adults, the gesture group spent significantly less time on problem solving than the no cue group, even though there was no significant difference found in learning achievements between the groups.

It is important to consider how any perceived increased engagement relates to an instructor's gesturing. Is it the social cues the instructor deliveres that prompts generative processing and makes the learner try to pay attention harder? Is it possible that it is not the instructor, but the gestures themselves that "may contribute to comprehension by capturing and maintaining listeners' attention" (Hostetter, 2011, p. 299)? Hostetter calls both of these interpretations into question since "gestures do not always enhance communication regardless of speaking topic, as would be predicted if gestures had their communicative effect through a general attention- or rapportbuilding mechanism" (p. 305). If gesturing is simply a matter of calling attention to the relevant area, should not non-human cueing serve a similar benefit? Two of the studies had a non-human cueing condition in addition to the gesture and no gesture group (Ouwehand et al., 2015b; Pi et al., 2017). Ouwehand et al. (2015b) found no difference between the cueing and gesturing groups for learning or cognitive load. Pi et al. (2017) found that the gesture groups showed better learning and attention than the cueing group, although there was no difference in cognitive load. Interestingly, there was no difference between the cueing and no cuing/gesturing group for learning, attention focus, and cognitive load (Pi et al., 2017). It is difficult to draw any conclusions from these somewhat contradictory results. One interesting point is that if gestures were processed automatically, as mentioned in the opening section on gestures, one might expect the gesture groups to experience a smaller cognitive load than the cueing group.

Listeners reported liking speakers who gesture more than those who do not (Kelly \& Goldsmith, 2004). In other words, the instructor becomes more emotionally interesting while gesturing. Does this indicate that the instructor's presence is essential for gestures to be effective? Koumoutsakis et al. (2016) had the model face away from the students in both the gesture and no gesture videos to control for any social cues caused by facial expressions/gaze. They found that, "the odds of solving a standard problem correctly after instruction that included speech and gesture were 28.82 times the odds after instruction that included only speech" (Koumoutsakis et al., 2016, p. 310). Carlson et al. (2014) compared a voice over video to the gesture condition that only included a hand and arm visible, but still found that the gesture group had significantly higher learning gains than the no gesture case. These experiments introduced factors that would seem to significantly limit the social cues normally available, but still found benefits from gestures. While more studies are needed, it still raises some interesting questions as to whether gestures gain benefits due to social cues or something inherent to the gestures themselves.

Fiorella and Mayer (2016) add an interesting layer to this discussion, though it focused on drawing and not gesturing. Similar to Carlson et al. (2014), Fiorella and Mayer (2016) found that a video with an arm drawing a diagram was more effective for transfer knowledge, as compared to the diagram being already drawn with the same explanation given. Furthermore, they found that a video drawing the diagram without a hand in place was not more effective than the video with the diagram already created. Interestingly, they also found that the arm only 
video was more effective than one with the instructor's body included. While not strictly gesturing, this study raises some very interesting questions about what the right mixture of social cues and instructor presence might be to facilitate learning.

\section{DISCUSSION}

Due to the diverse nature of the literature in video studies, there are limited comparators among studies and thus, broad trends are difficult to determine. It seems clear, however, that including an instructor as an onscreen agent significantly impacts the attention of students. Despite the suspected splitting of attention between the instructor and the media, there was no subsequent decrease in learning outcomes. The analysis gets even stranger when we look at the cognitive load. The limited number of studies based on self-reported data point to a conclusion that the instructor being on screen did not increase the cognitive load. This would seem to imply that something must be countering this split attention affect. Since these studies were not specifically utilizing gesturing by the instructor and in most cases, the videos were identical, except for the presence of the instructor, it seems likely to be a factor of the instructor presence itself. It is unclear if the cognitive load scales used would accurately measure an increase in generative processing, which may provide clues as to if students felt increased social cues or partnership with the instructor due to their presence on screen. Another possibility is that the interpretation of the onscreen agent is a biologically primary knowledge, which does not require extra cognitive processing, even with the increased attention. An alternate possibility could be postulated from the study by Kim et al (2014). They studied the viewing habits of students in MOOC courses involving 862 videos and specifically looked for peaks in times where people dropped out of watching a video. They found that $61 \%$ of these peaks occurred near a visual transition (such as going from an instructor video to a PowerPoint). Though a bit far-fetched, it seems possible that the presence of an instructor onscreen would moderate any visual transition, as one part of the screen is remaining the same. This may be worth further study. We can also contrast the results from the gesturing part of the review, as they also incorporate the instructor being on screen. In terms of cognitive load, the results were very limited, but again, seemed to support the premise that an onscreen agent does not cause an increase in cognitive load. There does seem to be support for the idea that gestures can support increased learning. A potential boundary condition would be that redundant pointing gestures or simple beat gestures are not effective.

One interesting question that emerged was whether gestures require the full instructor on screen to be effective or if they may even be more effective with just the arm or hand visible. This would be a potential downside to the use of the lightboard, as generally it involves the full instructor being on screen; however, videos could be redesigned to just include an arm or hand by using a smaller portion of the board. Overall, there is no conclusive evidence about whether lightboard videos will be effective, but there is reason to be optimistic. It seems likely that they will not increase cognitive load, even with the instructor on the screen. Utilizing gestures, as well as cueing via coloured markers, could be helpful in realizing the full potential of these videos. Of course, it would also be essential to utilize the established principles advocated in CTML, both during video creation, as well as during post production. There is a vast amount of work to be done with regard to the lightboard, but it seems like it could be beneficial.

\section{LIMITATIONS}

One significant limitation is that this literature review is meant to assist with determining the effectiveness of lightboard videos, but due to a lack of published research, it relied on tangentially related studies. Thus, the conclusions drawn have to be general in nature, but might still assist with guiding the design of lightboard videos for future testing.

\section{FUTURE RESEARCH}

There is a definitive need for empirical qualitative and quantitative research on the lightboard. At the moment, there appears to be no published work, so research of almost any type would be of value. Specifically, comparing the effectiveness of lightboard videos versus other types is of paramount importance. This should involve both experimental studies (i.e. having one group watch a lightboard video and another group watch an equivalent one) as well as studies in authentic classes. It would be important to measure a wide range of conditions, such as the use of gestures, comparing different types of gestures, the use of colour coding, the inclusion of various media, etc. In terms of variables, data on learning outcomes would likely be the easiest to obtain, but cognitive load and engagement/attention data would be useful to supplement it. It would be essential to determine the best approach or mix of approaches to measure this accurately. Coding survey and focus group responses would enrich the data and obtaining open-ended responses from learners might guide future research directions. 
It would also be interesting to study the social aspect of the instructor on screen. Would there be certain behaviours that could contribute to a personalization effect in these videos? Some possibilities might include comparing formal drawings versus quick sketches, looking at the camera versus looking at the board, or even perhaps formal versus informal clothing, as that seemed to be an area as yet unstudied.

\section{REFERENCES}

Bhat, Suma, Chinprutthiwong, P., \& Perry, M. (2015). Seeing the Instructor in Two Video Styles: Preferences and Patterns. International Educational Data Mining Society. Retrieved from https:/ / eric.ed.gov/?id=ED560520

Brame, C. J. (2016). Effective Educational Videos: Principles and Guidelines for Maximizing Student Learning from Video Content. CBE Life Sciences Education, 15(4). https:/ / doi.org/10.1187/cbe.16-03-0125

Butcher, K. (2014). The Multimedia Principle. In R. Mayer (Ed.), The Cambridge Handbook of Multimedia Learning (Cambridge Handbooks in Psychology, pp. 174-205). Cambridge: Cambridge University Press. https:// doi.org/10.1017/CBO9781139547369.010

Carlson, C., Jacobs, S. A., Perry, M., \& Breckinridge Church, R. (2014). The effect of gestured instruction on the learning of physical causality problems. Gesture, 14(1), 26-45. https:/ / doi.org/10.1075/gest.14.1.02car

Chen, C.-M., \& Wu, C.-H. (2015). Effects of different video lecture types on sustained attention, emotion, cognitive load, and learning performance. Computers $\mathcal{E}$ Education, 80, 108-121. https:/ / doi.org/10.1016/j.compedu.2014.08.015

Church, R. B., Ayman-Nolley, S., \& Mahootian, S. (2004). The Role of Gesture in Bilingual Education: Does Gesture Enhance Learning? International Journal of Bilingual Education and Bilingualism, 7(4), 303-319. https://doi.org/10.1080/13670050408667815

Cook, S. W., Duffy, R. G., \& Fenn, K. M. (2013). Consolidation and Transfer of Learning After Observing Hand Gesture. Child Development, 84(6), 1863-1871. https://doi.org/10.1111/cdev.12097

Dey, E. L., Burn, H. E., \& Gerdes, D. (2009). Bringing the Classroom to the Web: Effects of Using New Technologies to Capture and Deliver Lectures. Research in Higher Education, 50(4), 377-393. https:/ / doi.org/10.1007/s11162-009-9124-0

Fiorella, L., \& Mayer, R. E. (2016). Effects of Observing the Instructor Draw Diagrams on Learning from Multimedia Messages. Journal of Educational Psychology, 108(4), 528-546. https:/ / doi.org/10.1037/edu0000065

Fung, F. M. (2017). Adopting Lightboard for a Chemistry Flipped Classroom To Improve Technology-Enhanced Videos for Better Learner Engagement. Journal of Chemical Education, 94(7), 956-959. https:// doi.org/10.1021/acs.jchemed.7b00004

Ginns, P. (2005). Meta-analysis of the modality effect. Learning and Instruction, 15(4), 313-331. https:/ / doi.org/10.1016/j.learninstruc.2005.07.001

Guo, P. J., Kim, J., \& Rubin, R. (2014). How Video Production Affects Student Engagement: An Empirical Study of MOOC Videos. In Proceedings of the First ACM Conference on Learning @ Scale Conference (pp. 41-50). New York, NY, USA: ACM. https:/ / doi.org/10.1145/2556325.2566239

Hong, J., Pi, Z., \& Yang, J. (2018). Learning declarative and procedural knowledge via video lectures: cognitive load and learning effectiveness. Innovations in Education and Teaching International, 55(1), 74-81. https:/ / doi.org/10.1080/14703297.2016.1237371

Hoogerheide, V., Loyens, S. M. M., \& van Gog, T. (2014). Comparing the effects of worked examples and modeling examples on learning. Computers in Human Behavior, 41(Complete), 80-91. https:/ / doi.org/10.1016/j.chb.2014.09.013

Hostetter, A. B. (2011). When do gestures communicate? A meta-analysis. Psychological Bulletin, 137(2), $297-315$. https:// doi.org/10.1037/a0022128

Ilioudi, C., Giannakos, M., \& Chorianopoulos, K. (2013). Investigating Differences among the Commonly Used Video Lecture Styles. https:/ / doi.org/10.13140/2.1.3524.9284

Kang, S., Hallman, G., Son, L., \& Black, J. (2013). The Different Benefits from Different Gestures in Understanding a Concept. Journal of Science Education and Technology, 22(6), 825-837. https:/ / doi.org/10.1007/s10956-0129433-5

Kim, J., Guo, P. J., Seaton, D. T., Mitros, P., Gajos, K. Z., \& Miller, R. C. (2014). Understanding In-video Dropouts and Interaction Peaks Inonline Lecture Videos. In Proceedings of the First ACM Conference on Learning @ Scale Conference (pp. 31-40). New York, NY, USA: ACM. https:/ / doi.org/10.1145/2556325.2566237 
Kizilcec, R. F., Bailenson, J. N., \& Gomez, C. J. (2015). The instructor's face in video instruction: Evidence from two large-scale field studies. Journal of Educational Psychology, 107(3), 724-739. https:// doi.org/10.1037/edu0000013

Kizilcec, R. F., Papadopoulos, K., \& Sritanyaratana, L. (2014). Showing Face in Video Instruction: Effects on Information Retention, Visual Attention, and Affect. In Proceedings of the SIGCHI Conference on Human Factors in Computing Systems (pp. 2095-2102). New York, NY, USA: ACM. https:/ / doi.org/10.1145/2556288.2557207

Korving, H., Hernández, M., \& De Groot, E. (2016). Look at me and pay attention! A study on the relation between visibility and attention in weblectures. Computers $\mathcal{E}$ Education, 94, 151-161. https:/ / doi.org/10.1016/j.compedu.2015.11.011

Koumoutsakis, T., Church, R., Alibali, M., Singer, M., \& Ayman-Nolley, S. (2016). Gesture in Instruction: Evidence from Live and Video Lessons. Journal of Nonverbal Behavior, 40(4), 301-315. https:/ / doi.org/10.1007/s10919$016-0234-z$

Mayer, R. (2005). Cognitive Theory of Multimedia Learning. In R. Mayer (Ed.), The Cambridge Handbook of Multimedia Learning (Cambridge Handbooks in Psychology, pp. 31-48). Cambridge: Cambridge University Press. https:// doi.org/10.1017/CBO9780511816819.004

Mayer, R. (2014). Principles Based on Social Cues in Multimedia Learning: Personalization, Voice, Image, and Embodiment Principles. In R. Mayer (Ed.), The Cambridge Handbook of Multimedia Learning (Cambridge Handbooks in Psychology, pp. 345-368). Cambridge: Cambridge University Press. https:/ / doi.org/10.1017/CBO9781139547369.017

Mayer, R.E. (2017). Using multimedia for e-learning. Journal of Computer Assisted Learning, 33(5), $403-423$. https://doi.org/10.1111/jcal.12197

Mayer, Richard E., Heiser, J., \& Lonn, S. (2001). Cognitive constraints on multimedia learning: When presenting more material results in less understanding. Journal of Educational Psychology, 93(1), 187-198. https:/ / doi.org/10.1037/0022-0663.93.1.187

Mayer, Richard E., \& Moreno, R. (2003). Nine Ways to Reduce Cognitive Load in Multimedia Learning. Educational Psychologist, 38(1), 43-52. https:// doi.org/10.1207/S15326985EP3801_6

Moreno, R., Mayer, R. E., Spires, H. A., \& Lester, J. C. (2001). The Case for Social Agency in Computer-based Teaching: Do Students Learn More Deeply When They Interact with Animated Pedagogical Agents? Cognition and Instruction, 19(2), 177-213. https:/ / doi.org/10.1207/S1532690XCI1902_02

Ouwehand, K., van Gog, T., \& Paas, F. (2015a). Designing Effective Video-Based Modeling Examples Using Gaze and Gesture Cues. Journal of Educational Technology E Society, 18(4), 78-88.

Ouwehand, K., van Gog, T., \& Paas, F. (2015b). Effects of Gestures on Older Adults' Learning from Video-based Models. Applied Cognitive Psychology, 29(1), 115-128. https:/ / doi.org/10.1002/acp.3097

Ozan, O., \& Ozarslan, Y. (2016). Video lecture watching behaviors of learners in online courses. Educational Media International, 53(1), 27-41. https:/ / doi.org/10.1080/09523987.2016.1189255

Paas, F. (1992). Training strategies for attaining transfer of problem-solving skill in statistics: A cognitive-load approach. Journal of Educational Psychology, 84, 429-434. https:/ / doi.org/10.1037/0022-0663.84.4.429

Paas, F., \& Sweller, J. (2014). Implications of Cognitive Load Theory for Multimedia Learning. In R. Mayer (Ed.), The Cambridge Handbook of Multimedia Learning (Cambridge Handbooks in Psychology, pp. 27-42). Cambridge: Cambridge University Press. https:/ / doi.org/10.1017/CBO9781139547369.004

Pao-Ta Yu, Yuan-Hsun Liao, \& Ming-Hsiang Su. (2013). A Near-Reality Approach to Improve the e-Learning Open Courseware. Journal of Educational Technology \& Society, 16(4), 242-257.

$\mathrm{Pi}, \mathrm{Z}$., \& Hong, J. (2016). Learning process and learning outcomes of video podcasts including the instructor and PPT slides: a Chinese case. Innovations in Education $\mathcal{E}$ Teaching International, 53(2), 135-144. https:/ / doi.org/10.1080/14703297.2015.1060133

Pi, Z., Hong, J., \& Yang, J. (2017). Effects of the instructor's pointing gestures on learning performance in video lectures. British Journal of Educational Technology, 48(4), 1020-1029. https:/ / doi.org/10.1111/bjet.12471

Pierson, A. (2017). The Effect of Seeing an Instructor's Face within an Instructional Video on Connectedness, Attention, and Satisfaction (Ph.D.). Northcentral University, United States -- Arizona. Retrieved from http:/ / search.proquest.com/docview/1936016522/abstract/8DA626AE8DDD44EDPQ/1

Rueckert, L., Church, R. B., Avila, A., \& Trejo, T. (2017). Gesture enhances learning of a complex statistical concept. Cognitive Research: Principles and Implications, 2, 2. https:/ / doi.org/10.1186/s41235-016-0036-1 
Schneider, S., Beege, M., Nebel, S., \& Rey, G. D. (2018). A meta-analysis of how signaling affects learning with media. Educational Research Review, 23, 1-24. https:/ / doi.org/10.1016/j.edurev.2017.11.001

Skibinski, E. S., DeBenedetti, W. J. I., Ortoll-Bloch, A. G., \& Hines, M. A. (2015). A Blackboard for the 21st Century: An Inexpensive Light Board Projection System for Classroom Use. Journal of Chemical Education, 92(10), 17541756. https:// doi.org/10.1021/acs.jchemed.5b00155

Valenzeno, L., Alibali, M. W., \& Klatzky, R. (2003). Teachers' gestures facilitate students' learning: A lesson in symmetry. Contemporary Educational Psychology, 28(2), 187-204. https://doi.org/10.1016/S0361476X(02)00007-3

van der Meij, H. (2017). Reviews in instructional video. Computers \& Education, 114, 164-174. https://doi.org/10.1016/j.compedu.2017.07.002

van Gog, T., Verveer, I., \& Verveer, L. (2014). Learning from video modeling examples: Effects of seeing the human model's face. Computers $\mathcal{E}$ Education, 72, 323-327. https:// doi.org/10.1016/j.compedu.2013.12.004

van Wermeskerken, M., Ravensbergen, S., \& van Gog, T. (2017). Effects of instructor presence in video modeling examples on attention and learning. Computers in Human Behavior. https://doi.org/10.1016/j.chb.2017.11.038

van Wermeskerken, M., \& van Gog, T. (2017). Seeing the instructor's face and gaze in demonstration video examples affects attention allocation but not learning. Computers $\mathcal{E}$ Education, 113, 98-107. https://doi.org/10.1016/j.compedu.2017.05.013

Wang, J., \& Antonenko, P. D. (2017). Instructor presence in instructional video: Effects on visual attention, recall, and perceived learning. Computers in Human Behavior, 71, 79-89. https:/ / doi.org/10.1016/j.chb.2017.01.049

Wermeskerken, M. van, Grimmius, B., \& Gog, T. van. (2018). Attention to the model's face when learning from video modeling examples in adolescents with and without autism spectrum disorder. Journal of Computer Assisted Learning, 34(1), 32-41. https:/ / doi.org/10.1111/jcal.12211

Yeo, A., Ledesma, I., Nathan, M. J., Alibali, M. W., \& Church, R. B. (2017). Teachers' gestures and students' learning: sometimes "hands off" is better. Cognitive Research: Principles and Implications, 2, 41. https:/ / doi.org/10.1186/s41235-017-0077-0

Yousef, A. M. F., Schroeder, U., \& Chatti, M. A. (2014). Video-Based Learning: A Critical Analysis of The Research Published in 2003-2013 and Future Visions (No. RWTH-CONV-204082). IARIA. https:/ / doi.org/999910338682, 978-1-61208-328-5, 2308-4367

\section{http://www.ejmste.com}

